\title{
TV/Series
}

19 | 2021

Perfectionnisme et séries télévisées. Hommage à Stanley Cavell (1926-2018)

\section{Manhunt: Unabomber : conversation avec une cabane, une boîte aux lettres et un feu tricolore}

Laurence Espinosa

\section{OpenEdition}

\section{Journals}

Édition électronique

URL : https://journals.openedition.org/tvseries/5218

DOI : $10.4000 /$ tvseries. 5218

ISSN : 2266-0909

Éditeur

GRIC - Groupe de recherche Identités et Cultures

Référence électronique

Laurence Espinosa, « Manhunt: Unabomber : conversation avec une cabane, une boîte aux lettres et un feu tricolore », TV/Series [En ligne], 19 | 2021, mis en ligne le 06 mai 2021, consulté le 14 mai 2021 URL : http://journals.openedition.org/tvseries/5218; DOI : https://doi.org/10.4000/tvseries.5218

Ce document a été généré automatiquement le 14 mai 2021

\section{cc) (†)}

TV/Series est mis à disposition selon les termes de la licence Creative Commons Attribution - Pas d'Utilisation Commerciale - Pas de Modification 4.0 International. 


\title{
Manhunt : Unabomber : conversation avec une cabane, une boîte aux lettres et un feu tricolore
}

\author{
Laurence Espinosa
}

\begin{abstract}
" Où nous trouvons-nous? Dans une série dont nous ne connaissons pas les extrémités, et dont nous croyons qu'elle n'en a point ${ }^{1}$. "
\end{abstract}

\section{Introduction}

\section{Questionnement initial}

Comment dire la philosophie de Stanley Cavell que je suis encore en train de découvrir? Comment dire sa philosophie à partir d'une anthropologie ressentie comme titubante? Dans le propos qui suit, j'ai choisi d'aborder ces questionnements à partir d'une mini-série télévisée américaine de huit épisodes de quarante-deux minutes réalisée par Greg Yaltanes, créée par Andrew Sadrowski, Jim Clemente et Tony Gittelson, diffusée du $1^{\text {er }}$ août au 12 septembre 2017 sur Discovery Channel et accessible sur Netflix depuis le 12 décembre 2017 : Manhunt: Unabomber. Elle raconte une traque, la confrontation entre James R. Fitzgerald, un agent du FBI interprété par Sam Worthington et Ted Kaczynski, The Unabomber², un terroriste incarné par Paul Bettany.

\section{Aller vers}

2 J'ai regardé plusieurs fois la série. La première était pour me divertir, les autres pour la parcourir. J'ai appuyé sur les touches «pause», «retour en arrière» ou «accélération ». J'ai longuement visionné certaines séquences et j'ai fait parler aux 
personnages des langues différentes. J'ai peut-être fait ce que j'aimerais parfois faire avec le réel ; lui donner d'autres chances.

3 «Le terrain » est une notion qui tiraille mes recherches. Quand j'ai repris des études en anthropologie, je me sentais illégitime pour n'avoir à parcourir que des textes ou ne pas être partie vers un ailleurs géographiquement situé ${ }^{3}$. Pour me rassurer, je crois m'être d'abord hasardée du côté du postmodernisme de la Writing Culture, du terrain multisite de George Marcus ${ }^{4}$ et du néo modernisme revendiqué par Jean et John Comaroff ${ }^{5}$. Plus récemment, $\mathrm{j}$ 'ai eu accès aux travaux de Tim Ingold qui propose de dissocier l'ethnographie de l'anthropologie, deux disciplines qui auraient des ambitions différentes ${ }^{6}$. Ce dernier qui envisage l'anthropologie comme une éducation ${ }^{7}$ m'a permis d'esquisser un pont vers Stanley Cavell pour lequel le cinéma notamment hollywoodien est aussi une éducation et interroger le perfectionnisme moral ${ }^{8}$. Dans un autre élan, j'ai effleuré le travail sensible de Veena Das ou tenté de toucher la transformation du souffrir en s'ouvrir'.

4 Pour entamer la conversation avec ce monde autre, j'ai cherché des «compagnons obscurs » qui pourraient me frayer des passages dans les images ${ }^{10}$. J'en ai choisi trois : la cabane, la boite aux lettres et le feu tricolore. J'ai retenu ces personnages-objets comme étant les plus récurrents des huit épisodes. J'ai parfois triché un peu quand j'ai pris en compte des avatars (discutables) tels les courriers pour la boite aux lettres et la cellule ou la carcasse d'une voiture pour la cabane. L'exposé qui suit est composé des récits de ces informateurs.

5 La cabane suggère une entrée en matière, voire une entrée dans la matière, à savoir un entrelacement de suppositions et superpositions. La boite aux lettres, elle, semble proposer des repères tangibles. Elle est l'indice qui rappelle la raison de la traque, qui nomme les protagonistes de l'histoire et sert de support à l'enquête du FBI. Elle est un insert de certitude. Le feu tricolore dont le feu rouge remplit l'écran de la dernière scène telle un soleil artificiel et aveuglant dit l'interstice ou ce qui me revient : «Des souvenirs de cinéma se superposent fil à fil aux souvenirs de ma vie ${ }^{11}$ ».

\section{Récit 1 - La cabane - entrée en ou dans la matière}

6 Dans le huitième épisode, la cabane de Ted Kaczynski est soulevée, suspendue à un hélicoptère par un filin et posée sur un camion pour être transportée. Les passants et les téléspectateurs s'interrompent pour regarder cette scène insolite. Je considère alors la cabane comme un objet-personnage parce qu'elle semble avoir à dire.

Habitat du fugitif, elle est aussi l'abri provisoire de l'agent fédéral qui le poursuit et dans mes souvenirs, elle renvoie à Henry David Thoreau. Ces croisements ou superpositions seront donc les premiers éléments que j'interrogerai pour aller vers Stanley Cavell.

\subsection{Leurres}

8 Un homme hirsute marche dans les bois. Dans l'une de ses mains, il tient des lapins. Sur le chemin, il ramasse des morilles, écoute le chant des oiseaux et s'arrête pour se rafraichir dans un cours d'eau. Il vit dans une maisonnette (E01). La série est inspirée de faits réels et le téléspectateur averti peut penser qu'il est en présence de Ted 
Kaczynski (Ted) ${ }^{12}$. En fait, quelques scènes plus tard, il apprend que l'homme en question est James R. Fitzgerald (Fitz), un agent du FBI. D'ailleurs, si nous sommes attentifs, nous remarquons que sa cabane n'est pas dans le bois, mais à l'orée du bois. La démarcation est subtile, mais fait peut-être sens. Quoi qu'il en soit, dès cet instant, une forme de confusion s'installe épaissie par des réminiscences du récit de Henry David Thoreau sur son expérience de Walden ${ }^{13}$.

\subsection{Trouble}

9 Fitz poursuit Ted aux sens propre et figuré. Il le trouve «brillant, patient et méticuleux » et avoue à son frère, David: «Je le connais aussi bien que moi » (E05). Ainsi, avant le procès, il confie à Ted : « Je vous admirais tellement que j'ai tout plaqué pour vivre dans les bois comme vous » (E03). Il voudrait être Ted parce qu'il ne parvient pas à se trouver : "Sur quelle voie dois-je m'engager?", dit-il à la linguiste Natalie Rogers (E03). Ted, quand à lui, aimerait "éviscérer publiquement» (E08) cet hôte visiblement indésirable. Les deux personnages sont imbriqués induisant un trouble chez le téléspectateur.

\subsection{L'enjeu de la cabane}

10 Comme je l'ai signifié précédemment, les yeux fixés sur l'écran, je ne parviens pas toujours à me contenter de l'intrigue filmée. La scène de la cabane vient la complexifier. J'entends ainsi Henry David Thoreau qui raconte :

Quand j'écrivis les pages suivantes, ou plutôt en écrivis le principal, je vivais seul, dans les bois, à un mille de tout voisinage, en une maison que j'avais bâtie moimême, au bord de l'Étang de Walden, à Concord, Massachussetts, et ne devais ma vie qu'au travail de mes mains ${ }^{14}$.

11 Je me plais alors à penser que le fugitif et l'écrivain ne pourraient faire qu'un. La cabane de Ted a été « conçue selon un rapport mathématique parfait; la perfection dans la simplicité » (David, E05). «Cette cabane était un symbole de courage moral » (Fitz, E08). Henry David Thoreau a également choisi de vivre dans la simplicité :

Lorsque pour la première fois je fixai ma demeure dans les bois, c'est-à-dire commençai à y passer mes nuits aussi bien que mes jours, ce qui, par hasard, tomba le jour anniversaire de l'Indépendance, le 4 juillet 1845, ma maison, non terminée pour l'hiver, n'était qu'une simple protection contre la pluie, sans plâtrage ni cheminée, les murs en étant de planches raboteuses, passées au pinceau des intempéries, avec de larges fentes, ce qui la rendait fraîche la nuit. Les étais verticaux nouvellement taillés, la porte fraîchement rabotée et l'emplacement des fenêtres lui donnaient un air propre et aéré, surtout le matin, alors que la charpente en était saturée de rosée au point de me laisser croire que vers midi il en exsudait quelque gomme sucrée. Avec cet abri plus résistant autour de moi, j'avais fait quelque progrès pour ce qui est de se fixer dans le monde ${ }^{15}$.

12 Les deux situations peuvent-elles être confondues? Les auteurs de la série ne l'ont-ils pas voulu ainsi? Je me contente d'abord d'une analogie poussive. Je constate en effet, qu'à un peu plus d'un siècle de distance, les deux expériences ont duré deux ans. A partir du 4 juillet 1845 Henry David Thoreau s'est retiré dans les bois pour un peu plus de deux ans et la traque de Ted par Fitz s'est déroulée de 1995 à 1997. Le premier a choisi de s'extraire temporairement du monde pour protester contre une politique gouvernementale esclavagiste et impérialiste. Sa cabane est devenue l'embouchure 
d'un mégaphone susceptible de porter sa voix dans un souci de perfectibilité démocratique. La cabane de Ted apparait comme une carapace qui le protège et l'isole d'un monde qu'il juge hostile. En ce sens, la cellule où il purge encore aujourd'hui sa peine est une extension partielle de la cabane ${ }^{16}$. Les alarmes et les cliquetis des serrures ont remplacé les chants d'oiseaux, de cascades et le bruissement des feuilles. Mais, l'isolement est resté intact. Quand Henry David Thoreau est allé en prison pour avoir refusé de payer ses impôts, il n'y est resté qu'une nuit.

\subsection{Chassés croisés ou vers Stanley Cavell}

13 Stanley Cavell a écrit The Senses of Walden en écho au Walden de David Henry Thoreau ${ }^{17}$. Cette seule occurrence l'autoriserait à s'inviter dans la série. Quoi qu'il en soit, je choisis, à ce niveau de la réflexion de faire un gros plan sur les femmes, celles qui accompagnent Fitz et Ted. Courageuses et indépendantes, les femmes tracent leur propre chemin et pourrait donner à Manhunt : Unabomber quelque chose à voir avec À la recherche $d u$ bonheur ${ }^{18}$. Par ailleurs, les relations compliquées ou exacerbées de Ted et Fitz avec les femmes transforment, par moment, le thriller en mélodrame, ce qui nous mènerait plutôt vers Contesting tears ${ }^{19}$.

Au début de la série, Fitz est présenté comme un homme ordinaire, un Américain de la classe moyenne avec son pavillon de banlieue, ses trois enfants et son épouse Elly. Cette dernière a organisé une fête en son honneur car il a obtenu son diplôme de profiler de l'unité d'analyse comportementale de Quantico en Virginie. Fitz, agent de la brigade anti-graffiti, a suivi des cours du soir pour devenir « un homme respectable, faire partie de l'élite.» (Elly, E01). Sa famille est très fière de lui. Ce bonheur affiché est éphémère puisque le soir même de la fête, des agents du FBI débarquent pour demander à Fitz de l'aide sur une affaire qu'ils tardent à résoudre. À partir de cet instant, le nouveau profiler va progressivement s'éloigner de sa famille géographiquement et sentimentalement. Il va de moins en moins communiquer avec Elly et finir par placer ses enfants au second plan de ses préoccupations. Dans une scène, il déchire un dessin intitulé « Papa arrête les méchants » pour y inscrire une adresse utile à l'enquête (E04) et abandonne ses fils au cinéma pour aller récupérer un fax (E05).

$\mathrm{Au}$ cours de l'enquête, Fitz rencontre Natalie Rogers, une linguiste, qui va l'épauler dans les analyses des écrits de Ted. Quand Elly rencontre pour la première fois la jeune femme son regard trahit sa jalousie (E04). Cependant, lorsque Natalie tente d'embrasser Fitz, ce dernier la repousse. Elle imagine qu'elle n'est alors qu'une "simple auxiliaire pour se rapprocher de Ted » (E05). Ted est la seule affaire qui vaille, il n'en cherche pas d'autres (affair) ${ }^{20}$. Lorsque sa collègue Tabby, agent de terrain qui suit des cours du soir en psychologie, met en jeu sa carrière en interceptant un document qu'elle lui transmet illégalement, il la dénonce auprès de la hiérarchie et lui fait comprendre qu'elle est seule responsable de son geste. Elly, Natalie ou Tabby ne parviennent pas toujours à établir la conversation avec Fitz, mais elles ne perdent pas pied pour autant. C'est Fitz qui se perd tandis qu'elles poursuivent leurs chemins respectifs. Elles n'ont rien à attendre de lui.

16 Là où la conversation de Fitz avec les femmes est erratique et minimaliste, celle de Ted est inexistante ou presque. Pour lui, les femmes ne sont que des "gonzesses ${ }^{21}$. Plusieurs d'entre elles ont abîmé sa vie. Elles l'ont éloigné de ceux qui comptaient pour lui comme son ami d'enfance Doug avec qui il partageait une carcasse de voiture et son 
frère David avec qui il a construit la cabane (E06). C'est sa belle-sœur qui reconnait son style d'écriture dans le journal ; événement qui va conduire à son arrestation (E05). Il ne semble pas avoir su construire une relation amoureuse durable. Il en aurait entamé une avec Hélène qui se serait terminée par un flot de poèmes obscènes (E05). Cependant, lorsque nous le rencontrons dans une bibliothèque de Lincoln, il semble avoir noué de bonnes relations avec la responsable Theresa et son fils Tim. L'adolescent lui demande de l'aide pour résoudre des équations et lui parler de problèmes relationnels dans le cadre scolaire. En suivant, nous voyons Ted se rêver en époux et père de famille ou autrement dit se projeter dans une image sociale conforme (E06).

Le mélodrame apparait comme une dispersion de possibles et confronte à l'impossibilité, celle de faire couple dans le cas présent. La cabane pourrait dire la maison où se nouent ces intrigues secondaires mais en écho avec un quotidien des téléspectateurs.

\section{Récit 2 - La boîte aux lettres - Insert de certitudes}

18 Le premier épisode commence par un gros plan sur une boite aux lettres bleue de l'US Postal. La voix de Ted en off annonce que «l'US Mail est digne de réflexion»(E01). La cabane nous a confronté au doute en tant qu'ouverture sur des impasses et des possibilités. La boite aux lettres semble proposer un cadre à nos pensées éparpillées. Elle est omniprésente en tant que réceptacle métallique identifiable, mais aussi par ses avatars que sont les différents courriers. Elle semble nous rappeler ou faire en sorte que nous n'oubliions pas le cœur de l'intrigue: la traque par le FBI d'un terroriste qui atteint ses cibles par des colis piégés.

Stan Cole, l'un des enquêteurs de l'unité d'analyses comportementale ne croit qu'aux preuves matérielles. Il veut des certitudes. C'est pourquoi il se montre sceptique quant à l'efficacité des méthodes de Fitz qui expérimente la linguistique judiciaire. Que peut nous dire ce récit de Stanley Cavell?

\subsection{Enquêtes}

Les interrogations sur les conditions de l'enquête sont récurrentes en anthropologie. Même si elles ont atteint une certaine acuité dans la vision postmoderniste de la Writing Culture, elles ont existé dès les débuts de la discipline comme le souligne Jean Copans ${ }^{22}$. Évoquant la situation contemporaine avec les progrès de la cartographie et des statistiques, l'anthropologue se demande si nous n'assistons pas à la fin des pratiques de terrain telles qu'elles auraient été généralisées à partir des séjours de Bronislaw Malinowski (1884-1942). Ainsi, il ne s'agirait pas de programmer une mort de l'anthropologie, mais d'accompagner ou amener ses métamorphoses. Comme je l'ai souligné en introduction, Tim Ingold propose de séparer le processus ethnographique des spéculations anthropologiques. Dans le contexte qui nous préoccupe, comment mener l'enquête?

Dans une étude sur la bureaucratie, l'anthropologue David Graeber consacre un chapitre à la poste ${ }^{23}$. Je présente très brièvement son argumentation. Il commence par souligner que Max Weber s'est inspiré de la poste allemande pour décrire la bureaucratie en tant qu'incarnation de l'efficacité rationnelle. Ainsi, la poste aurait été l'une des premières tentatives d'appliquer des méthodes militaires à la sphère 
publique, à l'usage des commerçants d'abord, puis des particuliers. Efficace, elle devient un outil de présence accrue de l'État sur le territoire. David Graeber précise :

Et, effectivement, la Poste allemande de la fin du xixe siècle était vraiment impressionnante; elle pouvait se vanter d'effectuer jusqu'à cinq ou même neuf distributions par jour dans les grandes villes et s'enorgueillir, dans la capitale, d'un immense réseau de kilomètres de tubes pneumatiques conçus pour envoyer presque instantanément des lettres et de petits paquets sur une longue distance par un système d'air comprimé $e^{24}$. Cole, il faut essentiellement suivre la procédure c'est-à-dire rechercher des empreintes, des cheveux ou toute autre trace tangible. Le colis n'est pas piégé, mais FC (Fuck Computer puis Freedom Club) ${ }^{25}$ menace de faire exploser un avion de ligne à Los Angeles si son texte de 56 pages, La société industrielle et son futur n'est pas publiéé ${ }^{26}$.

La menace écartée, les policiers parviennent à établir une carte de distribution du courrier qui couvre toute la côte ouest des États-Unis. Le recoupement de ce schéma avec d'autres indices quantifiés permet de proposer un nom: Alan Meecks. Les enquêteurs pensent alors avoir résolu leur première affaire par ordinateur. Mais, c'est une fausse piste. L'homme était en prison au moment des faits (E02). Parallèlement à cette recherche factuelle, les psychologues du département ont établi un portrait, synthèse de dix ans d'enquête. Ainsi, le fugitif serait un mécanicien vivant dans la baie de San Francisco, aurait un faible QI et serait certainement impuissant. Stan Cole charge Fitz de rédiger un topo à partir de ces éléments pour mettre en évidence ce qu'il nomme le «complexe du gourdin» (E03). Fitz qui vient de commencer l'analyse des 
écrits de Ted, pense que ses collègues se trompent. L'homme recherché serait plutôt instruit et aurait plus de 50 ans. Il ne peut donc s'agir de Leo Frederik Burt, autre coupable pressenti par le FBI, qui est trop jeune (E03). Fitz suggère de publier le manifeste dans le Washington Post. Comme le journal n'est distribué que dans un seul point de vente sur le périmètre de référence, les acheteurs pourront être aisément surveillés. La procureure générale Janet Reno accepte le plan malgré sa mise à l'épreuve dans l'affaire Waco en 1993. L'opération de surveillance est un échec. Fitz est remercié et décrit comme « un connard, comme les autres. » (Ackermann, E04).

David interpelé par son épouse Laure prend contact avec l'avocat Antony Bisceglie. Celui-ci envoie une lettre de Ted au FBI afin qu'elle soit analysée. L'anonymat doit être respecté. Les agents constatent que la machine à écrire ne correspond pas et considèrent que Ted n'est pas Unabomber. Cependant, Tabby intercepte la lettre et en transmet une copie à Fitz qui s'écrie : « C'est lui » (E05).

Les premières images de Lincoln où réside le fugitif sont quatre boites aux lettres auprès desquelles le FBI va établir son campement. Les policiers débarquent dans le Montana comme employés des Telecom. Pour Stan Cole, il ne manque plus qu'un mandat et pour le procureur, il faut un motif raisonnable (E06 et E07).

\subsection{La voix inaudible de Ted}

29 Le manuscrit de 56 pages dont il est question dans la série parlerait de la société moderne et des technologies portant atteinte aux libertés humaines. J'y reviendrai. Pour le moment, je m'intéresserai aux mots qui sont au cœur de l'intrigue et qui suggèrent Stanley Cavell. Pour ce dernier, je l'ai déjà souligné, le cinéma est une éducation comme l'est l'anthropologie pour Tim Ingold. En ce sens, le cinéma et l'anthropologie disent des choses de la vie des humains, de leurs moments les plus ordinaires. Avant que Fitz ne soit contacté par le FBI, il est simplement en train de remplir une grille de mots croisés (E01). Cette séquence prépare le téléspectateur à celle qui suit. En effet, les agents demandent à Fitz impliqué dans l'univers des mots, d'analyser un document. Le profiler remarque alors que les lettres qui commencent chaque paragraphe forment un anacyclique : «D.A.D.I.T.I.S. - Dad it is » (E01) ${ }^{27}$. Il s'en amuse et en conclut qu'il s'agit certainement d'un clin d'œil de l'expéditeur. Loué pour sa perspicacité, il est invité à rejoindre l'unité «Unabom». Le terroriste vient de commettre un attentat. Il ne s'était pas manifesté depuis 6 ans.

30 Fitz qui part sur les lieux de l'explosion en déduit que les cibles sont plus des symboles que des personnes en particulier. Il s'adresse en off à l'expéditeur de la bombe: «Qu'essayez-vous de me dire?» (E01). Il déduit du document que Unabomber aime les énigmes et qu'il a des difficultés de communication (E02).

31 Les collègues de Fitz se moquent de son accent. Originaire de Philadelphie, il prononce wudder pour water. Le profiler réagit à la situation en faisant remarquer : «Il suffit d'un mot et vous savez tout sur moi » (E02). Le mot prononcé trahit les origines sociale et géographique de Fitz. Même si le téléspectateur peut entendre la voix de Ted dès le début de la série, les enquêteurs doivent se contenter d'écrits. Ted est muet et Fitz constate que personne n'écrit comme lui ou comme ça (E02).

Le manifeste est une succession de longues phrases. Il contient des notes, une bibliographie et une page de corrections. Le FBI contacte des experts dont certains sont cités dans le document pour approfondir son analyse. Une linguiste, Natalie Rogers, 
enseignante à Stanford, note que la présentation particulière du manuscrit correspond à la mise en page standard des thèses de doctorat d'avant $1972^{28}$. L'équipe se met alors à rechercher les idiolectes ou variantes orthographiques. Certaines d'entre elles suivent le guide stylistique du Chicago Tribune. Dans une autre scène du même épisode, dans un bar, Natalie décrit à Fitz des communautés slaves des zones marécageuses de Pripyat pour lui faire comprendre qu'il ne faut pas seulement se focaliser sur ce qui est dit mais aussi sur ce qui ne l'est pas. Dans le manifeste, $F C$ ne parle pas d'épouse, d'enfant, de famille, de travail, d'amis, d'ordinateur ou de télé. Fitz en déduit que le fugitif est éloigné de la culture populaire et n'a pas ou peu d'argent. C'est un marginal coupé de la société (E03).

Ted a écrit des centaines de lettres, notamment à sa mère et son frère David (E05). Après la publication de pages du manuscrit dans la presse, il envoie une lettre à son frère pour lui annoncer qu'il va arrêter une "activité particulière qui n'est plus nécessaire " (E06). Il témoigne alors d'un profond aveu de scepticisme: «J'ai eu l'impression de regarder le monde à travers une fenêtre » (E06). Stanley Cavell s'est inspiré des travaux sur le langage de John Langshaw Austin et Ludwig Wittgenstein. Pour ce dernier, le monde ordinaire est le foyer du langage ordinaire. Il faut essayer de dire les choses telles qu'elles sont ${ }^{29}$. Les aveux de Ted confirment que son retrait au monde est, comme pressenti dans le récit de la cabane, le signe d'une incapacité, celle de sa mutité. Quand Theresa s'exclame: "La revendication nous parle " (E05), elle ouvre la porte au claim exploré par Stanley Cavell comme liant démocratique ${ }^{30}$. En d'autres termes, Ted n'a pas de voix.

Cent agents du FBI sont désormais déployés à Lincoln. Il leur faut un mandat pour intervenir et celui-ci ne sera rédigé qu'à partir d'une preuve irréfutable. Cette preuve est un proverbe. Ted ne dit pas : "You can't have your cake and eat it too", mais " you can't eat your cake and have it too » ou dans la version française: «On ne peut pas avoir le beurre et l'argent du beurre ", mais " On ne peut pas avoir l'argent du beurre et le beurre » (E08). Le langage de Ted n'est pas ordinaire et c'est en ce sens qu'il n'arrive pas à converser. Il se réfère à la forme la moins usitée du proverbe comme pour le prendre à rebours ou ne savoir comment sortir du compte à rebours qui va couvrir sa voix d'un seul boum!

\section{Récit 3 - Le feu tricolore : ce qui me revient}

Avant chaque épisode, un bandeau rappelle que la série est inspirée de faits réels. Alors, je tiens à avertir le lecteur que, dans cette étude, je parle de personnages dont Ted Kaczynski incarné par Paul Bettany. Ceci explique que je n'ai pas lu le manifeste de 56 pages qui n'est qu'une image.

Ce sont des mots fragmentés qui atteignent les cibles de Unabomber. Que dit alors la série de l'idéologie de ce dernier qui d'après son frère a le « courage de vivre selon ses idéaux » (E05) ? Comme lui-même en convient, Ted est un terroriste (E07). Ce mot ne peut que me faire vaciller après les attentats emblématiques de Charlie Hebdo et du Bataclan perpétrés respectivement les 7 janvier et 13 novembre 2015 en France. Comment ces événements ont-ils altéré mon ordinaire ? Cette interrogation m'amène à reprendre un fil de discussion antérieur où je parcourais la guerre, la maladie, le terrorisme et le fait de se battre. 
37 J'aime le personnage qui danse sous la pluie près de sa cabane en écoutant de la musique classique (E06), mais les interférences d'un réel m'empêchent de communier avec cette scène extatique.

\subsection{Fragments} interlocuteurs. La cabane serait la trace à la fois trop visible et estompée de l'univers transcendantal de Henry David Thoreau. Elle devient, sans un soupçon d'intervention magique, la trame élémentaire dont Stanley Cavell se saisit pour l'étirer vers les possibles dans The Senses of Walden. Il est encore question de l'écrivain de Concord dans le récit du feu tricolore qui parle d'obéissance et de désobéissance. Á diverses reprises, Stan Cole dit à Fitz: "Tout ce qu'on vous demande, c'est obéir ». Ces injonctions répétées n'empêchent pas Fitz de mener l'enquête à sa façon. Il désobéit régulièrement aux ordres de ses supérieurs. Après avoir discuté avec Ted sur les méfaits de la société industrielle qui transforme les humains en " moutons $\|^{33}$, Fitz se retrouve en arrêt à un feu rouge alors qu'il n'y a aucun autre véhicule sur la route. La caméra se focalise sur son regard dubitatif. Qu'est-ce qui l'empêche d'avancer (E03) ? En écho à cette scène, son emménagement à l'orée du bois, à l'issue de son licenciement après que l'enquête piétine, peut-il être comparé à l'exil délibérément temporaire de Henry David Thoreau ? Pour ce dernier la désobéissance est un non qui doit faire écho ${ }^{34}$. Fitz à la lisière du bois, résistant aux injonctions de Cole, admirant parfois Ted, délaissant sa famille, avance par à-coups. Ses soubresauts seraient-ils des réponses aux fragments de Ted ou des échos qui dans le temps de la série ne peuvent que se rétracter?

41 Ted voulait prévenir ses contemporains des dangers de la société industrielle ou de technologies de plus en plus envahissantes et coercitives. Le cri d'alarme aurait été pour lui l'imitation par un oiseau d'une sirène de voiture (E05). Tabby, la collègue de Fitz, acquiesce : « La technologie ça craint » (E02). à un professeur de Harvard sans doute affilié à la CIA, Henry Murray, la «main de Dieu » (E06) $)^{35}$. Pour le psychologue, les idées de Ted sont «peu originales, stéréotypées, absurdes », en bref des « régurgitations de Jacques Ellul » (E06).

43

Les cliquetis des claviers, les sirènes, les serrures, les moteurs sont le fond sonore dominant de la série. Ce brouhaha n'est momentanément interrompu que par le chant des oiseaux ou des cascades et le bruissement des feuilles ou par les explosions des colis piégés. 


\subsection{Se battre} avec intérêt des extraits de son manifeste dans le journal : "Tu soutiens un terroriste maintenant? » (E06). Il est un terroriste parce que pour lui : « Le seul moyen de nous libérer sera de tout faire exploser » (E02). Cependant, malgré ces aveux, il regrette cette image qu'il renvoie de lui : «Je serai un islamiste dès les débuts du procès » (E08). Si avant 1997, il est possible de mentionner l'attentat contre le World Trade Center du 26 février 1993 perpétré par quatre islamistes intégristes, la plupart des attentats de cette période sont le fait de mouvances d'extrême droite comme en Arizona le 9 octobre 1995, à Oklahoma City le 19 avril 1995 ou à Atlanta le 27 juillet 1996. Il est alors pertinent de se demander si la remarque de Ted n'est pas plus en rapport avec le contexte de réception de la série qu'avec celui de l'intrigue. Les paroles rapportées des autorités à ce propos renforcent cette perception: "Le gouvernement américain ne négocie pas avec les terroristes » (Cole, E04), ou RENO. « Quand il s'agit de terrorisme, on trouve toujours un moyen de trouver du temps» (Reno, E04), ou encore «On ne s'abaisse pas à négocier avec un terroriste » (journaliste, E04). Le terrorisme apparait dans ces paroles comme l'urgence primordiale. Le USA Patriot Act date cependant du 26 octobre 2001.

L'ordinaire est sans doute ce quotidien auquel nous prêtons peu d'attention, de la même façon que nous accordons peu d'intérêt à la vie en train de se faire. Le langage ordinaire est aussi un inaperçu. L'acte terroriste, dans sa soudaine brutalité, semble rompre ce fil. Mais est-ce parce qu'il est hors de l'ordinaire ou parce qu'il nous jette l'ordinaire à la face? Pour tenter de nous éclairer, Stanley Cavell continue la conversation de et avec Henry David Thoreau dans Senses of Walden et emprunte ainsi la voie ouverte par Rallph Waldo Emerson ${ }^{36}$. Il ne s'agit pas d'imiter ou de commenter le texte originel mais de l'augmenter en partant de soi. Le perfectionnisme moral implique avant toute chose de se faire confiance (self reliance) : «Croire votre pensée, croire que ce qui est vrai pour vous dans l'intimité de votre cœur est vrai pour tous les hommes - c'est là le génie ${ }^{37} »$. Pour autant, cette volonté d'amélioration de soi n'est pas un repli sur soi. Elle est aussi une courroie vers le perfectionnement du monde.

En 2016, j'ai été invitée à participer à un séminaire par une association de lutte contre les addictions. Je devais m'interroger sur le religieux comme potentielle addiction. Prenant en compte l'actualité du moment, j'ai consacré l'une des parties de cet exposé aux jeunes Occidentaux partis rejoindre le dit État islamique. Reprenant la définition de Claude Olivenstein qui décrivait l'addiction comme la rencontre entre un produit, une personnalité et un moment socio-culturel ${ }^{38}$, je me suis demandé dans quelle mesure ces jeunes n'étaient pas comparables à des drogués. Dans cette perspective, j'ai retenu une conception extrême de la religion comme produit, la mondialisation et ses frontières brouillées comme contexte et le sentiment de ne pas exister pour ce monde comme trait de personnalité. Jusqu'aux attentats de Charlie Hebdo, la majorité des jeunes Français ayant rejoint l'État islamique étaient considérés comme socialement et économiquement exclus ${ }^{39}$. Ils étaient majoritairement issus de classes populaires déstabilisées par des mutations sociales rapides; des minorités visibles confrontées au racisme et en quête d'une cause à défendre qui a pris la forme d'une guerre contre la société occidentale sous l'étendard d'un islam radicalement réinterprété. 

soin à l'hôpital. À l'abri de ma chambre, J'ai appris que des gens se rassemblaient spontanément dans les villes pour dire leur stupéfaction. J'étais triste de ne pas pouvoir être avec eux.

Le mot "guérir» vient de l'allemand wehren (défendre) ${ }^{40}$. Etymologiquement, il a quelque chose à voir avec la guerre et la sonorité du mot en renforce l'idée. Si guérir est "rendre la santé ${ }^{41}$, cela signifie que celle-ci a été prise. En ce sens, la santé est présentée comme une extériorité qui renvoie à l'extériorité de la maladie. Cet endehors n'est pas appréhendé comme bienfaisant, il est soupçonné d'être étranger et ennemi. Guérir revient à faire la guerre à la maladie.

Pour François Laplantine, les sociétés occidentales contemporaines admettent deux représentations majeures de la maladie et de la guérison.

La maladie est considérée comme entité exogène pénétrée par effraction dans le corps d'un individu qui n'y est pour rien, et la guérison consiste dans la jugulation allopathique d'une positivité ennemie avec laquelle il ne faut pas composer, mais qu'il faut littéralement anéantir. Inversement la maladie n'est plus perçue comme une entité étrangère au malade. Elle vient de lui et a une fonction valorisée ou tout au moins signifiante qui doit être décryptée comme une de ses composantes, et la guérison consiste alors dans une activité régulatrice qui n'est plus cette fois antagoniste aux symptômes du malade ${ }^{42}$.

L'auteur souligne que la première approche est la plus partagée par les malades et les médecins qui considèrent le symptôme comme un "ennemi à abattre » et la maladie comme une altérité. La seconde la voit en tant qu'altération et suggère que le malade soit son propre thérapeute. Quand j'ai annoncé à mes proches que j'étais atteinte d'une maladie incurable, ils ont été plusieurs à m'inviter à me battre. Si je pouvais entendre ces paroles bienveillantes, la formulation me mettait mal à l'aise. Contre qui ou quoi devrais-je me battre? Cette malade n'était-ce pas moi et donc pourquoi devais-je imaginer mon propre anéantissement? Je me suis alors souvenue que le discours ordinaire est ponctué d'injonctions à se battre pour trouver un travail, un logement ou se dépasser pour dépasser les autres parce qu'il faut se battre contre quelque chose ou quelqu'un. Ces ordres explicites ou implicites s'adressent le plus souvent aux plus fragiles ou à ceux qui sont en train de perdre et a fortiori se perdre. Je me suis demandé en suivant si ces jeunes qui avaient sans doute été confrontés à l'impératif de se battre pour sortir de leur condition, n'avaient pas fini par prendre l'expression au pied de la lettre pour donner une réponse réelle à leur désarroi en devenant de vrais combattants, avec de vraies armes qui feraient de vraies victimes. Quand Ted, avant le début du procès, prend conscience que sa folie meurtrière risque de l'enfermer dans la folie en tant que maladie mentale, il décide de plaider coupable. Il constate: « Tout ce qui me reste à faire c'est arrêter de me battre » (E08).

\section{Conclusion}

51 Fitz pris de doute dans son enquête dit : « J'espère ne pas être trop à côté de la plaque " (E03). C'est aussi la question que je me pose en écrivant ces mots de conclusion. J'ai voulu converser avec une série, par l'intermédiaire de personnages-objets pour qu'ils disent Stanley Cavell. J'ai laissé affleurer des idées à partir de relations amoureuses au goût de mélodrame, d'un langage estropié et du perfectionnisme dévoyé qu'implique l'injonction à se battre. Dans l'exposé sur les addictions, je pensais à la reconnaissance 
d'un humain désarmé, fragile et vulnérable. À se battre, j'ai opposé le dépôt des armes comme alternative. Les discussions en cours avec Manhunt : unabomber et Stanley Cavell m'enjoignent à altérer se battre pour en extraire dé-battre; une ondulation de la trame.

\section{BIBLIOGRAPHIE}

CAVELL, Stanley, À la recherche du bonheur. Hollywood et la comédie du remariage [1éd. : 1981], trad. franç. par Chistian FOURNIER et Sandra LAUGIER, Paris, Éditions de l'étoile/Cahiers du cinéma, coll. « Essais », 1993.

CAVELL, Stanley, Les voix de la raison. Wittgenstein, le scepticisme, la moralité et la tragédie [ $1{ }^{\mathrm{e}}$ éd. : 1979], trad. franç. par Sandra LAUGIER et Nicole BALSo, Paris, Seuil, coll. « L'ordre philosophique », 1996.

CAVELL, Stanley, Contesting Tears: The Hollywood Melodrama of the Unknown Woman, Chicago, University of Chicago Press, 1996.

CAVELL, Stanley, La projection du monde. Réflexions sur l'ontologie du cinéma [1 éd. : 1971], trad. franç. par Chistian FOURNIER, Paris, Belin, 1999.

CAVELL, Stanley, Sens de Walden [1 $1^{\mathrm{e}}$ éd. : 1972], trad. franç. par Bernard RIVAL et Omar BERRADA, Courbevoie, Théâtre typographique, 2007.

CAVELL, Stanley, Qu'est-ce que la philosophie américaine? De Wittgenstein à Emerson, trad. franç. par Christian FOURNIER et Sandra LAUGIER, Paris, Gallimard, coll. « Folio Essais », 2009.

CAVELL, Stanley, Philosophie des salles obscures. Lettres pédagogiques sur le registre de la vie morale [1 ${ }^{\mathrm{e}}$ éd. : 2004], trad. franç. par Nathalie FERRON, Mathias GIREL et Élise DOMENACH, Paris, Flammarion, coll. «Bibliothèque des savoirs », 2011.

CHRÉTIEN, Jean-Pierre, « Les premiers voyageurs étrangers au Burundi et au Rwanda : les "compagnons obscurs" des "explorateurs" ", Afrique \& Histoire, vol. 4, n 2, 2005, p. 37-72, https:// www.cairn.info/revue-afrique-et-histoire-2005-2-page-37.htm.

CLIFFORD, James, MARCUS, George, Writing Culture. The Poetics and Politics of Ethnogrphy, Oakland (CA), University of California Press, 1986.

COMAROFF, John and Jean, Ethnography and the historical imagination, Oxford, Westview Press, 1992. COPANS, Jean, "Vulnérabilités des situations d'enquête, des anthropologues et de leurs informateurs, ou des disciplines en sciences sociales? ", Anthropologie \& développement, $\mathrm{n}^{\circ}$ 42-43, 2015, p. 227-235, http://journals.openedition.org/anthropodev/379.

DAS, Veena, Life and words: violence and the descent into the ordinary, Oakland (CA), University of California Press, 2007.

DEJEAN, Mathieu, « La CIA a-t-elle vraiment "fabriqué" Unabomber ? », site des Inrockuptibles, 3 janvier 2018, https://www.lesinrocks.com/actu/la-cia-t-elle-vraiment-fabriqueunabomber-128312-03-01-2018/. 
EMERSON, Ralph Waldo, Essais, introduction et trad. franç. par Anne wiCKE, Paris, Michel Hourdiard, 2010.

EMERSON, Ralph Waldo, «Thoreau » [1 $1^{\mathrm{e}}$ éd.: 1862], in Henry David THOREAU, Walden, trad. franç. par Jacques MAILHOS, Paris, Gallmeister, 2017, p. 365-389.

GRAEBER, David, Bureaucratie, trad. franç. par Françoise et Paul CHEMLA, Paris, Les Liens qui libèrent, 2015.

INGOLD, Tim, Une brève histoire des lignes [ $1^{\mathrm{e}}$ éd. : 2007], trad. franç. par Sophie RENAUT, Bruxelles, Zones sensibles, 2011.

INGOLD, Tim, « Anthropology contra ethnography », HAU: Journal of Ethnographic Theory, vol. 7, $\mathrm{n}^{\circ} 1$, Spring 2017, p. 21-27, https://www.journals.uchicago.edu/doi/10.14318/hau7.1.005.

INGOLD, Tim, L'anthropologie comme éducation [1 $1^{\mathrm{e}}$ éd. : 2017], postface d'Yves CITTON, trad. franç. par Maryline PINTON, Rennes, Presses Universitaires de Rennes, 2018.

KHOSROKHAVARD, Fahrad, Radicalisation, Paris, Maison des Sciences de l'Homme, 2014.

LAPLANTINE, François, « Jalons pour une anthropologie des systèmes de représentations de la maladie et de la guérison dans les sociétés occidentales contemporaines ", Histoire, économie \& société, vol. 3, n 4, 1984, p. 641-650, https://www.persee.fr/doc/

hes_0752-5702_1984_num_3_4_1383.

olivenstein, Claude, Il n'y a pas de drogués heureux, Paris, Robert Laffont, coll. « Vécu », 1976.

THOREAU, Henry David, La désobéissance civile [1 $1^{\mathrm{e}}$ éd. : 1849], trad. franç. et postface de Guillaume VILLENEUVE, Paris, Éditions Mille et une nuits, 1996.

THOREAU, Henry David, Walden ou la vie dans les bois [1 $1^{\mathrm{e}}$ éd. : 1854], trad. franç. par Louis FABULET, Paris, Gallimard, coll. « L'Imaginaire », 2017.

UNABOMBER, Manifeste : l'avenir de la société industrielle, trad. franç. et présentation par Jean-Marie APOSTOLIDÈs, préface d'Annie LE BRUN, Paris, Jean-Jacques Pauvert aux Éditions du Rocher, 1996.

\section{NOTES}

1. Ralph Waldo Emerson, Expérience [1 ${ }^{\mathrm{e}}$ éd. : 1844], in Stanley Cavell, Qu'est-ce que la philosophie américaine, Paris, Gallimard, 2009, p. 504

2. Unabomber : surnom donné à Ted Kaczynski par le FBI et la presse pour UNiversity and Airline BOMber. Entre 1978 et 1985 le terroriste a envoyé 16 colis piégés destinés à des universitaires, des vendeurs d'ordinateurs ou des patrons de compagnie aérienne. Il a tué trois personnes et en a blessé vingt-trois.

3. Les écrits de missionnaires de la Société des missions évangéliques de Paris ont été les archives à partir desquelles j'ai étudié les Sotho au XIX ${ }^{\mathrm{e}}$ siècle. Je ne suis pas allée sur le terrain en tant qu'entité géographique.

4. James Clifford and George Marcus, Writing Culture. The Poetics and Politics of Ethnogrphy, Oakland (CA), University of California Press, 1986.

5. John and Jean Comaroff, Ethnography and the historical imagination, Oxford, Westview Press, 1992 ; pour faire le lien avec les travaux du sociologue américain Charles Wright Mills.

6. Tim Ingold, « Anthropology contra ethnography », HAU: Journal of Ethnographic Theory, vol. 7, n 1, Spring 2017, p. 21-27, https://www.journals.uchicago.edu/doi/10.14318/hau7.1.005. 
7. Tim Ingold, L'anthropologie comme éducation [1 $1^{\mathrm{e}}$ éd. : 2017], Rennes, Presses Universitaires de Rennes, 2018.

8. Stanley Cavell, Philosophie des salles obscures. Lettres pédagogiques sur le registre de la vie morale [1 $\mathrm{e}^{\mathrm{e}}$ éd. : 2004], Paris, Flammarion, coll. « Bibliothèque des savoirs ", 2011.

9. Veena Das, Life and words: violence and the descent into the ordinary, Oakland (CA), University of California Press, 2007.

10. Jean-Pierre Chrétien, «Les premiers voyageurs étrangers au Burundi et au Rwanda: les "compagnons obscurs" des "explorateurs" ", Afrique \& Histoire, vol. 4, n² 2, 2005, p. 37-72, https:// www.cairn.info/revue-afrique-et-histoire-2005-2-page-37.htm.

11. Stanley Cavell, La projection du monde. Réflexions sur l'ontologie du cinéma [1e éd. : 1971], Paris, Belin, 1999, p. 17.

12. Le 3 avril 1996, le FBI a arrêté Ted Kaczynski. Son arrestation a été très médiatisée.

13. Henry David Thoreau, Walden ou la vie dans les bois [1 ${ }^{\mathrm{e}}$ éd. : 1854], Paris, Gallimard, coll. « L'Imaginaire », 2017.

14. Ibid., p. 90

15. Ibid.

16. Ted Kaczynski est emprisonné à l'ADX Florence dans le Colorado, une prison de haute sécurité (Supermax).

17. Stanley Cavell, Sens de Walden [1 éd. : 1972], Courbevoie, Théâtre typographique, 2007.

18. Stanley Cavell, À la recherche du bonheur. Hollywood et la comédie du remariage [1 ${ }^{\mathrm{e}}$ éd. : 1981], Paris, Éditions de l'étoile/Cahiers du cinéma, coll. « Essais », 1993.

19. Stanley Cavell, Contesting Tears: The Hollywood Melodrama of the Unknown Woman, Chicago, University of Chicago Press, 1996.

20. Affair, affaire ou liaison, aventure

21. C'est ainsi que Ted parle des femmes dans son manuscrit: Chick.

22. Jean Copans, «Vulnérabilités des situations d'enquête, des anthropologues et de leurs informateurs, ou des disciplines en sciences sociales?", Anthropologie \& développement, $\mathrm{n}^{\circ} 42-43$, 2015, p. 227-235, http://journals.openedition.org/anthropodev/379.

23. David Graeber, Chap. 3 : «L'utopie des règles, ou pourquoi nous adorons la bureaucratie, au fond », in Bureaucratie, Paris, Les Liens qui libèrent, 2015, p. 175-241.

24. Ibid., p. 183.

25. Ces initiales qui se trouvent sur les constituants des bombes est le nom que se donne le fugitif. Les policiers le nomment Unabomber. J'ai choisi de l'appeler par son prénom, Ted.

26. Unabomber, Manifeste : l'avenir de la société industrielle, Paris, Jean-Jacques Pauvert aux Éditions du Rocher, 1996.

27. J'ai choisi de me référer à la version française de la série pour la plupart des dialogues. J'ai conservé la version originale pour les jeux de mots ou expression. De même, les ouvrages de références sont majoritairement des traductions, sauf quand ils n'ont pas été traduit ou que la version originale s'imposait.

28. Ted Kaczynski a soutenu une thèse en mathématique en 1969.

29. Voir Stanley Cavell, Les voix de la raison. Wittgenstein, le scepticisme, la moralité et la tragédie [1 ${ }^{\mathrm{e}}$ éd. : 1979], Paris, Seuil, coll. « L'ordre philosophique », 1996.

30. Ibid.

31. Tim Ingold, Une brève histoire des lignes [1 $1^{\mathrm{e}}$ éd. : 2007], Bruxelles, Zones sensibles, 2011.

32. "Trame", définition du Trésor de la langue française (TLF), http://atilf.atilf.fr/dendien/ scripts/tlfiv5/visusel.exe?29;s=3893871420;r=2;nat=;sol=2;.

33. Ted, par sa voix off, compare ses contemporains à des « moutons » (sheeps).

34. Henry David Thoreau, La désobéissance civile [1 ${ }^{\mathrm{e}}$ éd. : 1849], Paris, Éditions Mille et une nuits, 1996. 
35. Mathieu Dejean, « La CIA a-t-elle vraiment "fabriqué" Unabomber ? », site des Inrockuptibles, 3 janvier 2018, https://www.lesinrocks.com/actu/la-cia-t-elle-vraiment-fabriqueunabomber-128312-03-01-2018/.

36. Ralph Waldo Emerson, "Thoreau » [1 $1^{\mathrm{e}}$ éd. : 1862], in Henry David Thoreau, Walden, Paris, Gallmeister, 2017, p. 365-389.

37. Ralph Waldo Emerson, Essais, Paris, Michel Hourdiard, 2010, p. 29.

38. Claude Olivenstein, Il n'y a pas de drogués heureux, Paris, Robert Laffont, coll. « Vécu », 1976.

39. Fahrad Khosrokhavar, Radicalisation, Paris, Maison des Sciences de l'Homme, 2014.

40. « Guérir », TLF, http://atilf.atilf.fr/dendien/scripts/tlfiv5/advanced.exe?8;s=551535165;.

41. Ibid.

42. François Laplantine, "Jalons pour une anthropologie des systèmes de représentations de la maladie et de la guérison dans les sociétés occidentales contemporaines ", Histoire, économie \& société, vol. 3, $\mathrm{n}^{\circ} \quad 4, \quad 1984, \quad$ p. 641-650, https://www.persee.fr/doc/ hes_0752-5702_1984_num_3_4_1383, p. 642.

\section{RÉSUMÉS}

Les séries TV peuvent devenir des «terrains » au sens anthropologique, dans un dialogue avec la philosophie de Stanley Cavell. Et ces terrains engagent la subjectivité du chercheur comme les autres terrains anthropologiques. Ainsi la série Manhunt: Unabomber dit Cavell dans ses mots, ses images et ses sons. La cabane, la boite aux lettres et le feu tricolore, personnages - objets, disent Henry David Thoreau, le scepticisme, le langage ordinaire, la voix ou le perfectionnisme moral. L'écran nous rapproche et nous perd au rythme de la conversation qui affleure.

TV series can become "fields" in the anthropological sense, in a dialogue with Stanley Cavell's philosophy. And these fields engage the subjectivity of the researcher like the other anthropological fields. So The Manhunt: Unabomber series tells Stanley Cavell in its words, its images and its sounds. The cabin, the mailbox and the tricolor fire, object - characters, say Henry David Thoreau, skepticism, ordinary language, voice or moral perfectionism. The screen brings us closer and loses us to the rhythm of the conversation that comes out.

\section{INDEX}

Keywords : anthropology, conversation, language, perfectionism, terrorism

Mots-clés : anthropologie, conversation, langage, perfectionnisme, terrorisme

\section{AUTEUR}

\section{LAURENCE ESPINOSA}

Laurence Espinosa est docteure en anthropologie et chercheuse associée laboratoire ITEM (Identités, Territoires, Expressions, Mobilités, EA 3002, Université de Pau et des Pays de l'Adour). Elle intervient à l'Institut du travail social Pierre Bourdieu de Pau pour faire découvrir différents 
questionnements de l'anthropologie contemporaine. Elle est notamment l'auteure de : « La frontière : contrainte ou ressource pour l'individu ? Le "pari romantique" des pionniers de la Société des missions évangéliques de Paris, de "l'humanisation" à "l'humanitarisation" des Sotho » (Les Cahiers de Framespa, $\mathrm{n}^{\circ}$ 11, 2012, https://journals.openedition.org/framespa/2075). Elle a aussi mené des recherches sur le catholicisme en Pays Grenadois. Elle s'interroge en particulier aujourd'hui sur la notion de « terrain » en anthropologie.

Laurence Espinosa holds a Ph.D. in anthropology and is an associate researcher in the laboratory ITEM (Identities, Territories, Expressions, Mobility, EA 3002, University of Pau and Pays de l'Adour). She intervenes at the Institute of Social Work Pierre Bourdieu of Pau to discover different questions of contemporary anthropology. She is the author of : « La frontière : contrainte ou ressource pour l'individu ? Le "pari romantique" des pionniers de la Société des missions évangéliques de Paris, de "l'humanisation" à "l'humanitarisation" des Sotho » (Les Cahiers de Framespa, $\mathrm{n}^{\circ}$ 11, 2012, https://journals.openedition.org/framespa/2075). She has also conducted research on Catholicism in the Pays Grenadois. She questions in particular today the notion of "field" in anthropology. 